\title{
Relationship between alteration of the peptide hormone levels and depression during the gestational period
}

\author{
Keiichi Hiramoto ${ }^{1,2^{*}}$, Yurika Yamate ${ }^{2}$ and Hiromi Kobayashi ${ }^{2}$ \\ *Correspondence: hiramoto@suzuka-u.ac.jp

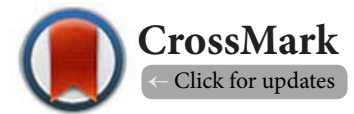 \\ 'Department of Pharmaceutical Science, Suzuka University of Medical Science, 3500-3 Minamitamagakicho, Suzuka, \\ Mie 513-8670, Japan. \\ 2Department of Dermatology, Osaka City University Graduate School of Medicine, 1-4-3 Asahimachi, Abeno, Osaka 545-8585, Japan.
}

\begin{abstract}
Background: Stress is considered to be a major factor in the development of depressive illness. Pregnancy is also a kind of physical stress, and the rate of pregnancy-induced depression is increasing. This study analyzed alterationsin the levels of peptide hormones in the gestational period in order to evaluate the prevalence of a depressed state during pregnancy.

Methods: Specific pathogen-free C57BL/6j gestation female mice were used for the experiments. In order to investigate the prevalence of a depressed state in the gestational period, we conducted forced swim test (FST) and open field test (OFT) and measured the levels of plasma adrenocorticotropic hormone (ACTH), $\beta$-endorphin ( $\beta$-End), corticosterone and dopamine (DA) in the gestational period. In addition, we measured the expression of prohormone convertase 2 (PC2) in the pituitary gland.

Results: In the FST, the akinesia time (floating time) during the gestational period was the longest on gestational day (gd) 6, then gradually decreasd toward parturition. On the other hand, in the OFT, the motor activity during the gestational period increased gradually to gd 17 after being the lowest on gd 6. The plasma levels of ACTH and corticosterone fell gradually to gd 18 after a peak on gd 6. In contrast, the plasma levels of $\beta$-End and DA and the expression of PC2 in the pituitary gland increased throughout the gestational period, peaking by gd 18 .

Conclusions: These observations suggest that the levels of ACTH and corticosterone change in parallel with those of $\beta$-End and DA during the gestational period and function to regulate a depressed state.
\end{abstract}

Keywords: Depression, $\beta$-Endorphin, adrenocorticotropic hormone, dopamine, forced swim test, open field test

\section{Introduction}

Adrenocorticotropic hormone (ACTH) is a peptide hormone of the hypothalamic-pituitary-adrenal axis (HPA axis) organs that is induced by various stressors following the stimulation of corticotropin-releasing hormone (CRH). The plasma concentrations of ACTH and $\mathrm{CRH}$ are maintained at high levels during pregnancy. The maternal secretion of pituitary ACTH and the subsequent plasma ACTH levels rise during pregnancy, while remaining within the normal limits, paralleling the rise in the plasma cortisol levels $[1,2]$. This rise in the maternal ACTH levels is due to circulating unbound placental $\mathrm{CRH}$ [3]. The ACTH concentration in amniotic fluid increases during pregnancy, peaking at the beginning of the third trimester, then exhibiting a decline [4].

On the other hand, depression is a common mental disorder that manifests as a depressed mood with loss of interest or pleasure, feelings of guilt or low self-worth, disturbed sleep or appetite, low energy and poor concentration. The prevalence of depressive symptoms during the gestational period has increased in recent years. Stress is considered to be a major factor in the development of depressive illness [5]. Many of the physiological and behavioral responses associated with stress are induced by $\mathrm{CRH}$. CRH has also been found to play a role in depressive illness [6].

Therefore, in this study, we evaluated the incidence of depressive symptoms during the gestational periodin mice and investigated the association between the HPA axis and depressive symptoms.

\section{Materials and methods \\ Animals}

Pregnant C57BL/6j mice (SLC, Hamamatsu, Japan) were housed individually on gestational day (gd) 0 to 18 of pregnancy. Pregnancy was determined by the observation of a vaginal plug. The plug date was considered to be gd 0 of gestation. The mice were allowed ad libitum access to food and water, and the 12-hour light/12-hour dark cycle, temperature $\left(23^{\circ} \mathrm{C}\right)$ and humidity (55\%) were kept constant. The animals were subjected to experiments according to the animal care regulations of Suzuka University of Medical Science. The mice were divided into 18 groups $(n=6)$.

\section{Forced swim test (FST, floating test)}

The mice were introduced to a transparent pool $\left(20 \times 35 \times 15 \mathrm{~cm}^{3}\right)$ 
Hiramoto et al. Research Journal of Endocrinology and Metabolism 2014,

filled with warm water $\left(30^{\circ} \mathrm{C}\right.$, height $\left.9.5 \mathrm{~cm}\right)$ and subjected to forced swimming for six minutes. A video camera recorded the experiment for six minutes. Then, we observed the behavior of the animals and measured the duration of complete immobility of the entire body for four minutes during the second half of the experiment.

\section{Open field test (OFT)}

The open field area $\left(50 \times 50 \times 40 \mathrm{~cm}^{3}\right)$ was made of plastic. The motor activity of the mice was measured over a 15-minute period using a video-tracking system (Smart2, Bio Research Center, Nagoya, Japan).

Analysis of the levels of peptide hormones, corticosterone and dopamine using an enzyme-linked immunosorbent assay (ELISA)

Blood samples were obtained from the heart on each day of pregnancy, and the plasma samples were fractionated. The plasma levels of $A C T H, \beta$-endorphin ( $\beta$-End), corticosterone and dopamine (DA) were determined using a commercial ELISA kits (ACTH and $\beta$-End; Phenix Pharmaceuticals Inc., CA; corticosterone; Assaypro, MO; DA; Abnova, Taipei, Taiwan) according to the manufacturer's instructions.

\section{Preparation and staining of the pituitary samples}

The pituitary specimens were fixed in phosphate-buffered paraformaldehyde (4\%), embedded in frozen Tissue-Tek, OCT compound and cut into 5 -mm-thick sections. The sections of the pituitary gland were washed in PBS and then subsequently incubated overnight at $4^{\circ} \mathrm{C}$ with rabbit anti-prohormone convertase 2 (PC2; 1:100) polyclonal antibodies (Santa Cruz Biotechnology Inc., Santa Cruz, CA), in order to determine the expression of PC2. The sections were then washed in PBS and incubated at room temperature for two hours with FITC-conjugated anti-mouse immunoglobulin (1:30; Dako Cytomation, Glostrup, Denmark). The expression levels of PC2 were evaluated immunohistochemically using a fluorescent microscope.

\section{Statistical analysis}

All data are presented as the mean \pm SD of results derived from six animals. The results for the two animal groups were analyzed with either Student's $t$-test or ANOVA usinga computer software package. Differences were considered to be significant for $p<0.05$.

\section{Results}

Effects of pregnancy on the mouse behavior during the FST

The FST is an examination that measures a depressed state. After being placed into the vessel containing water, the mice initially swim intensely to escape from the water, but then gradually give up and exhibit akinesia (immobility). In Figure 1, the duration of akinesia in the gestational period was the

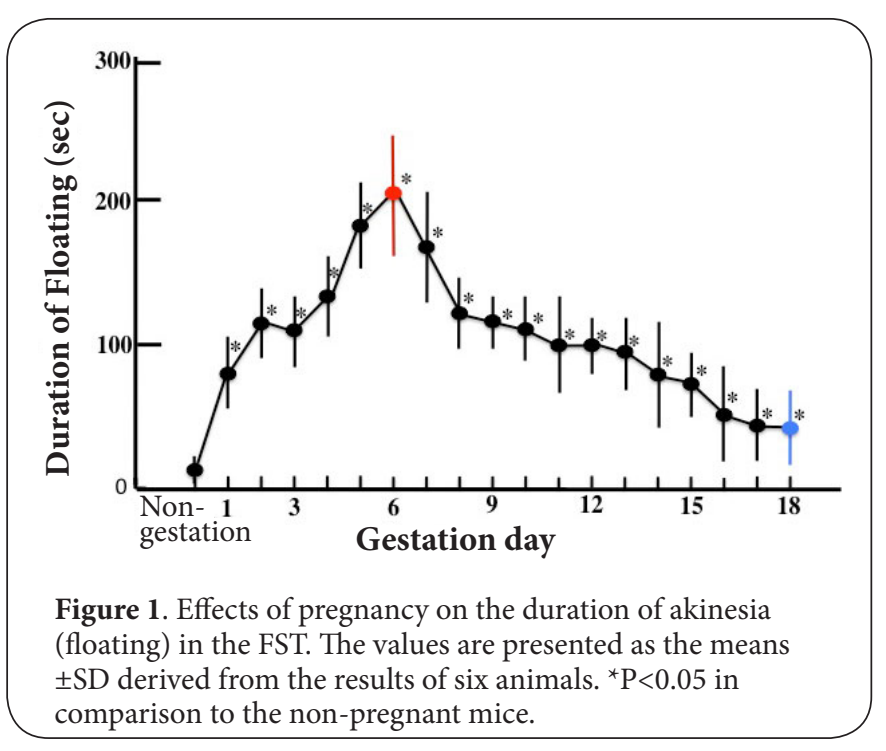

longest on gestational day (gd) 6 , then decreased gradually toward parturition.

Effects of pregnancy on the mouse behavior in the OFT It has been reported that a depressed state decreases the motor activity [7]. As shown in Figure 2, the motor activity during the gestational period gradually increased to gd 17 after reaching the lowest level on gd 6 .

Effects of pregnancy on the plasma levels of ACTH, $\beta$-End, corticosterone and DA

We measured the plasma levels of $\mathrm{ACTH}, \beta$-End, corticosterone and DA in the gestational period. The levels of ACTH (Figure 3A) and corticosterone (Figure 3B) fell gradually to gd 18 after

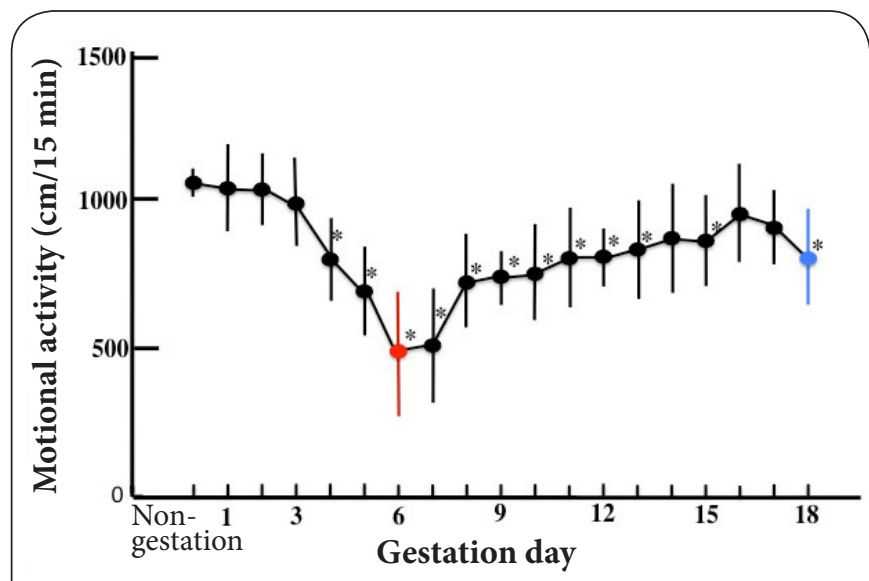

Figure 2. Effects of pregnancy on the duration of asthenia in the OFT. The values are presented as the means \pm SD derived from the results of six animals. ${ }^{\star} \mathrm{P}<0.05$ in comparison to the non-pregnant mice. 
exhibiting a peak on gd 6 . In contrast, the levels of $\beta$-End (Figure $3 \mathrm{~A}$ ) and DA (Figure $3 \mathrm{C}$ ) increased throughout the gestational period, peaking by gd 18 .
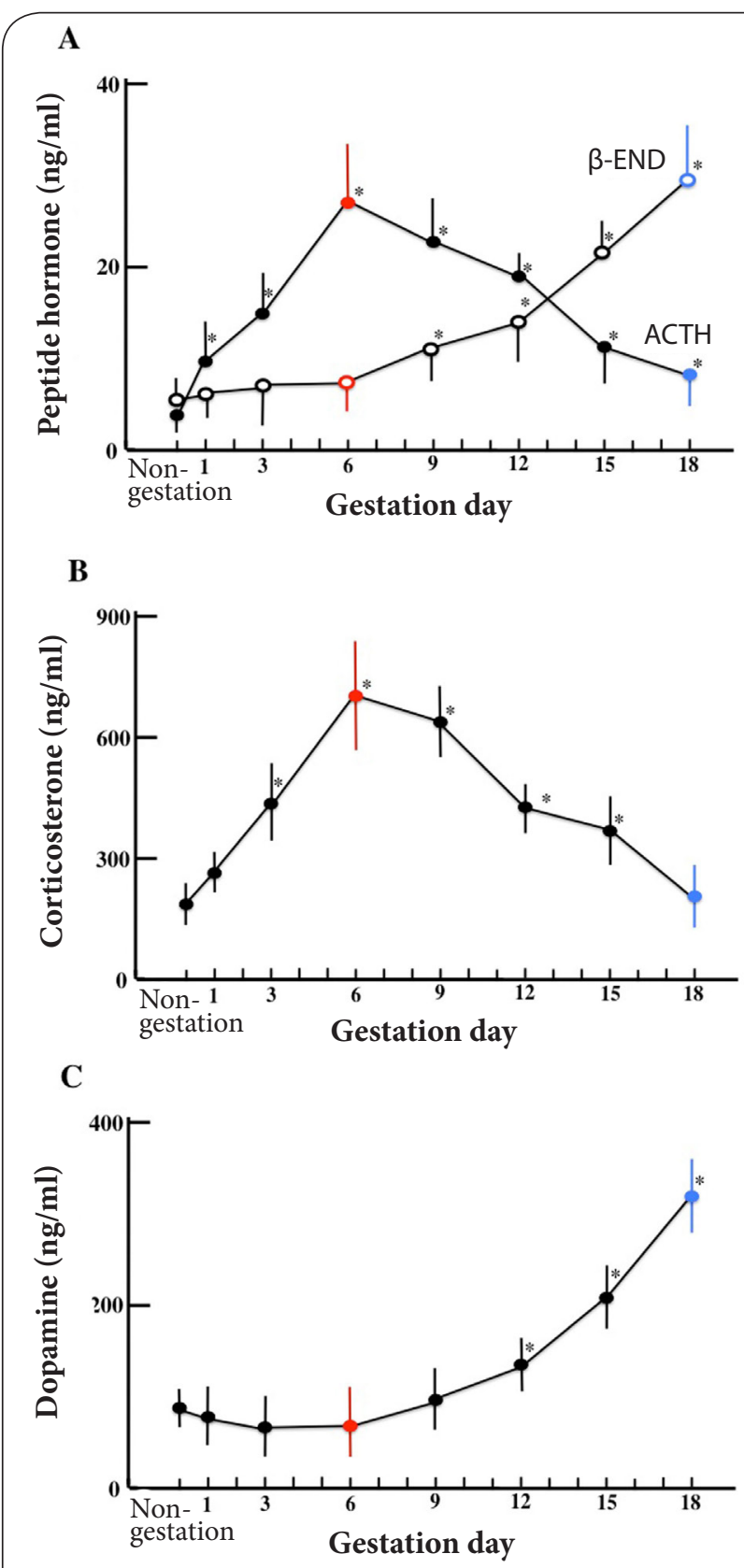

Figure 3. Effects of pregnancy on the plasma levels of ACTH, $\beta$-End (A), corticosterone (B), and DA (C). The values are presented as the means \pm SD derived from the results of six animals. ${ }^{*} \mathrm{P}<0.05$ in comparison to the non-pregnant mice.

Effects of pregnancy on the expression of PC2 in the pituitary gland

The production of the peptide hormones, ACTH, a-MSH, and $\beta$-End requires the proteolytic processing of proopiomelanocortin, which is hypothesized to utilize dual cysteine and subtilisin-like protease pathways, including the secretory vesicle cathepsin L pathway and PC pathway. In this study, the expression of PC2 increased gradually to gd 18 , with a peak on gd 18 (Figure 4).

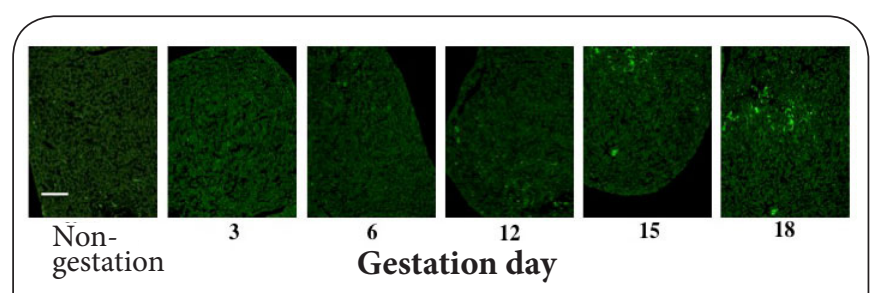

Figure 4. Effects of pregnancy on the expression of PC2 in the pituitary gland. The data reflect the results of typical experimentsin six animals. Scale bar $=10 \mu \mathrm{m}$.

\section{Discussion}

The present work demonstrated that the duration of akinesia on the FST was the longest at gd 6 and that a reduced level of motor activity was observed during the OFT. Furthermore, the levels of ACTH and corticosterone peaked on $\mathrm{gd} 6$, while the levels of $\beta$-End and DA peaked on gd 18.

Females are more susceptible to depression during pregnancy [8]. Pregnancy has been explored from the immunological point of view, since it can be considered a semi-allograft situation [9]. In this context, locally produced embryonic and endometrial $\mathrm{CRH}$ plays a role in both the aseptic inflammatory process of implantation and the antirejection process that protects the fetus from the maternal immune system [10]. Early in pregnancy, the implantation sites in the rat endometrium contain 3.5-fold higher concentrations of $\mathrm{CRH}$ compared to that observed in the inter-implantation regions. The increase in $\mathrm{CRH}$ stimulates the secretion of ACTH from the pituitary gland. In addition, the increase in the plasma ACTH levels occurs in parallel to the rise in the total corticosterone levels. In this study, the ACTH and corticosterone concentrations in the plasma increased during pregnancy, peaking at gd 6 , and exhibiting a decline. Dysregulation of the HPA axis characterized by glucocorticoid negative feedback resistance is frequently observed in human depressives [11]. Additionally, dysfunction of the dopaminergic and serotonergic systems in the prefrontal cortex (PFC) is thought to be involved in the development of a depressive state [11]. In rats, chronic stress induces a behaviorally depressive state, concomitant with dysregulation of the HPA axis and reductions in dopaminergic transmissions in the PFC [12]. From these reports, the presence of hypokinesis at the time of a psychical stress led to deviations in the dopaminergic neuron transmission due to the deficit of dopamine. Moreover, the cortisol level is adjusted based on the dopaminergic neuron transmission, 
and the increase in the cortisol level at the time of a psychical stress causes hypokinesis. Therefore, when a pregnant mouse is exposed to psychological stress, in this study, the akinesia time was longest on gd 6 , suggesting that malfunction of the dopaminergic system due to excess corticosterone was the cause. By gd 6, the ACTH/corticosterone system was considered to have contributed to the behavior strongly as opposed to the dopaminergic system. In these results, we propose that the $\mathrm{ACTH} /$ corticosterone system is concerned with the suppression of behavior under pregnancy and that the dopaminergic system is concerned with the activity of a behavior. Since it occurred during the gestational period, alterations of female hormones may be implicated; this issue is currently under examination. It is still not clear why this phenomenon occurred at that specific time (gd 6). In addition, in this study, we have measured corticosterone levels. In humans, the activity of cortisol is the highest of all glucocorticoids. However, in mice, the level of cortisol is very low, and corticosterone takes the place of cortisol. Therefore, we measured the corticosterone levels in the mice.

On the other hand, unlike ACTH, $\beta$-End increased gradually during the gestational period, exhibiting a peak by gd 18 . The neuronal network is modulated by opioids at the level of DA neurons and afferent structures, typically by the activation of opioid receptor enhancing reward- and motivation-related processes $[13,14]$. Therefore, we suggest that the $\beta$-End levels could be related to the activation of the dopaminergic system and that the activation of behaviors could be attributed to the $\beta$-End levels. Although the plasma levels of $\beta$-End increased at the end of pregnancy, the results indicated that this phenomenon is based on the increase in $\mathrm{PC2}$, which cleaves $\beta$-End from POMC in the pituitary gland (Figure 4). There is another cause of an increase in the $\beta$-End level, in addition to the high expression of PC2 in the pituitary gland. The levels of macrophages/monocytes, granulocytes, and lymphocytes increase during the gestational period $[15,16]$, with the levels of leukocytesin particular increasing at the end of pregnancy [17]. Thus, macrophages/monocytes, granulocytes and lymphocytes all secrete $\beta$-End. In addition, POMC, PC1, and PC2 are present in leukocytes, and POMC and its product, $\beta$-End, are apparently stored and released from secretory granules, similar to that observed in the decreased pathway in the pituitary gland [18]. However, the molecular mechanisms by which $P C 2$ increases during the late phase of pregnancyare unknown. Thus, two hormones, cortisol (corticosterone) and $\beta$-End, act at specific gestational ages, and they may have a role in maintaining the pregnancy.

In this study, on gd 6, the akinesia time was long,indicating a depressed state. Moreover, on gd 18, $\beta$-End was highly secreted and the gestation mice became active. According to the concept of Darwinian fitness, a spiritless state, which, is one of the symptoms of depression that prevents the consumption of energy required for an immune response. In addition, the spiritless state also allows the mother to recover by not moving and remaining still [19].

Therefore, a depressed state in a gestational age may be advantageous for maintaining a state of graviditas, and thereby improving the survival of the fetus. Our results therefore suggest that negative influences on the condition of the fetus can be avoided by suppressing the behavior of the mother's body on the most active day (gd 6) of embryonic differentiation. Conversely, this phenomenon is convenient for uplifting the mood during the intrapartum period (gd 18) and preparing for parturition. Furthermore, the level of IL-10 was highest on gd 6 (data not shown). Therefore, during the differentiation period, it is difficult to induce aggression of the immunological response to the fetus. Therefore,our results suggest that the peptide hormone-catecholamine-immune system protects the mother's body and the fetus from many risks during the gestational period.

\section{List of abbreviations}

FST: Forced swim test

OFT: Open field test

ACTH: Adrenocorticotropic hormone

$\beta$-End: $\beta$-Endorphin

DA: Dopamine

PC2: Prohormone convertase 2

gd: Gestational day

\section{Competing interests}

The authors declare that they have no competing interests.

Authors' contributions

\begin{tabular}{|l|c|c|c|}
\hline Authors' contributions & KH & YY & HK \\
\hline Research concept and design & $\checkmark$ & -- & $\checkmark$ \\
\hline Collection and/or assembly of data & $\checkmark$ & $\checkmark$ & -- \\
\hline Data analysis and interpretation & $\checkmark$ & $\checkmark$ & -- \\
\hline Writing the article & $\checkmark$ & -- & -- \\
\hline Critical revision of the article & $\checkmark$ & -- & $\checkmark$ \\
\hline Final approval of article & $\checkmark$ & $\checkmark$ & $\checkmark$ \\
\hline Statistical analysis & $\checkmark$ & -- & -- \\
\hline
\end{tabular}

Publication history

EIC: Jack Ronald Wall, University of Sydney, Australia.

Received: 08-Oct-2013 Revised: 25-Dec-2013

Accepted: 30-Jan-2014 Published: 05-Feb-2014

\section{References}

1. Sasaki A, Shinkawa O, Margioris AN, Liotta AS, Sato S, Murakami O, Go $M$, Shimizu $Y$, Hanew K and Yoshinaga K. Immunoreactive corticotropinreleasing hormone in human plasma during pregnancy, labor, and delivery. J Clin Endocrinol Metab. 1987; 64:224-9. | Article | PubMed

2. Laatikainen T, Virtanen T, Raisanen I and Salminen K. Immunoreactive corticotropin-releasing factor and corticotropin during pregnancy, labor and puerperium. Neuropeptides. 1987; 10:343-53. | Article | PubMed

3. Jeske W, Soszynski P, Rogozinski W, Lukaszewicz E, Latoszewska W and Snochowska H. Plasma GHRH, CRH, ACTH, beta-endorphin, human placental lactogen, $\mathrm{GH}$ and cortisol concentrations at the third trimester of pregnancy. Acta Endocrinol (Copenh). 1989; 120:785-9. | 
Hiramoto et al. Research Journal of Endocrinology and Metabolism 2014, http://www.hoajonline.com/journals/pdf/2053-3640-2-1.pdf

Article | PubMed

4. Tuimala R, Kauppila A and Haapalahti J. ACTH levels in amniotic fluid during pregnancy. Br J Obstet Gynaecol. 1976; 83:853-6. | Article | PubMed

5. Van Praag HM. Can stress cause depression. Prog. Neuropsychophamacol. Biol. Psychiatry. 2004; 28:891-907. | Article

6. Arborelius L, Owens MJ, Plotsky PM and Nemeroff CB. The role of corticotropin-releasing factor in depression and anxiety disorders. $J$ Endocrinol. 1999; 160:1-12. | Article | PubMed

7. Reneric JP and Lucki I. Antidepressant behavioral effects by dual inhibition of monoamine reuptake in the rat forced swimming test. Psychopharmacology (Berl). 1998; 136:190-7. | Article | PubMed

8. Timur $\mathrm{S}$ and Sahin $\mathrm{NH}$. The prevalence of depression symptoms and influencing factors among perimenopausal and postmenopausal women. Menopause. 2010; 17:545-51. | Article | PubMed

9. Makrigiannakis A, Zoumakis E, Kalantaridou S, Coutifaris C, Margioris AN, Coukos G, Rice KC, Gravanis A and Chrousos GP. Corticotropin-releasing hormone promotes blastocyst implantation and early maternal tolerance. Nat Immunol. 2001; 2:1018-24. | Article | PubMed

10. Mastorakos G, Scopa CD, Kao LC, Vryonidou A, Friedman TC, Kattis D, Phenekos C, Rabin D and Chrousos GP. Presence of immunoreactive corticotropin-releasing hormone in human endometrium. J Clin Endocrinol Metab. 1996; 81:1046-50. | Article | PubMed

11. Nestler EJ, Barrot M, DiLeone RJ, Eisch AJ, Gold SJ and Monteggia LM. Neurobiology of depression. Neuron. 2002; 34:13-25. | Article | PubMed

12. Mizoguchi K, Shoji H, Ikeda R, Tanaka Y and Tabira T. Persistent depressive state after chronic stress in rats is accompanied by HPA axis dysregulation and reduced prefrontal dopaminergic neurotransmission. Pharmacol Biochem Behav. 2008; 91:170-5. | Article | PubMed

13. Forbes EE, Hariri AR, Martin SL, Silk JS, Moyles DL, Fisher PM, Brown SM, Ryan ND, Birmaher B, Axelson DA and Dahl RE. Altered striatal activation predicting real-world positive affect in adolescent major depressive disorder. Am J Psychiatry. 2009; 166:64-73. | Article | PubMed Abstract I PubMed Full Text

14. Jutkiewicz EM and Roques BP. Endogenous opioids as physiological antidepressants: complementary role of delta receptors and dopamine. Neuropsychopharmacology. 2012; 37:303-4. | Article | PubMed Abstract I PubMed Full Text

15. De M, Choudhuri R and Wood GW. Determination of the number and distribution of macrophages, lymphocytes, and granulocytes in the mouse uterus from mating through implantation. J Leukoc Biol. 1991 50:252-62. | Article | PubMed

16. De M and Wood GW. Analysis of the number and distribution of macrophages, lymphocytes, and granulocytes in the mouse uterus from implantation through parturition. J Leukoc Biol. 1991; 50:381-92. | Article | PubMed

17. Matsumoto M, Sakao $Y$ and Akira S. Inducible expression of nuclear factor IL-6 increases endogenous gene expression of macrophage inflammatory protein-1 alpha, osteopontin and CD14 in a monocytic leukemia cell line. Int Immunol. 1998; 10:1825-35. | Article | PubMed

18. Mousa SA, Shakibaei M, Sitte N, Schafer M and Stein C. Subcellular pathways of beta-endorphin synthesis, processing, and release from immunocytes in inflammatory pain. Endocrinology. 2004; 145:1331-41. | Article | PubMed

19. Raison $\mathrm{CL}$ and Miller AH. The evolutionary significance of depression in Pathogen Host Defense (PATHOS-D). Mol Psychiatry. 2013; 18:15-37. | Article | PubMed Abstract | PubMed Full Text

\section{Citation:}

Hiramoto K, Yamate Y and Kobayashi H. Relationship between alteration of the peptide hormone levels and depression during the gestational period. Res $J$ Endocrinol Metab. 2014; 2:1.

http://dx.doi.org/10.7243/2053-3640-2-1 